\title{
On the symmetry of the distribution of $k$-crossings and $k$-nestings in graphs
}

\author{
Anna de Mier* \\ Submitted: Oct 11, 2006; Accepted: Nov 7, 2006; Published: Nov 23, 2006 \\ Mathematics Subject Classification: 05A19
}

\begin{abstract}
This note contains two results on the distribution of $k$-crossings and $k$-nestings in graphs. On the positive side, we exhibit a class of graphs for which there are as many $k$-noncrossing 2-nonnesting graphs as $k$-nonnesting 2-noncrossing graphs. This class consists of the graphs on $[n]$ where each vertex $x$ is joined to at most one vertex $y$ with $y<x$. On the negative side, we show that this is not the case if we consider arbitrary graphs. The counterexample is given in terms of fillings of Ferrers diagrams and solves a problem of Krattenthaler.
\end{abstract}

\section{Introduction}

Let $G$ be a graph on $[n]=\{1,2, \ldots, n\}$, with multiple edges allowed but without loops or isolated vertices. We say that the edges $\left\{i_{1}, j_{1}\right\}, \ldots,\left\{i_{k}, j_{k}\right\}$ are a $k$-crossing if $i_{1}<i_{2}<$ $\cdots<i_{k}<j_{1}<\cdots<j_{k}$, and that they are a $k$-nesting if $i_{1}<i_{2}<\cdots<i_{k}<j_{k}<\cdots<$ $j_{1}$. A graph with no $k$-crossing is called $k$-noncrossing and a graph with no $k$-nesting is called $k$-nonnesting. The largest $k$ for which a graph $G$ has a $k$-crossing (respectively, a $k$-nesting) is denoted $\operatorname{cros}(G)$ (resp., nest $(G))$.

The left-right degree sequence of a graph on $[n]$ is the sequence $\left(\left(l_{i}, r_{i}\right)\right)_{1 \leq i \leq n}$, where $l_{i}$ (resp., $r_{i}$ ) is the left (resp., right) degree of vertex $i$; by the left (resp., right) degree of $i$ we mean the number of edges that join $i$ to a vertex $j$ with $j<i$ (resp., $j>i$ ).

Chen et al.[1] show that the pair $(\operatorname{cros}(G)$, nest $(G))$ is symmetrically distributed among the set of graphs whose degree sequence is a fixed element of $\{(0,0),(0,1),(1,0),(1,1)\}^{n}$. Recently it has been shown (see [3]) that for each degree sequence $D$ there are as many graphs with $\operatorname{cros}(G) \leq k$ as with nest $(G) \leq k$. The purpose of this note is to explore to which extent the result of Chen et al. can be generalized to other classes of graphs. On the positive side, we show that when restricting left degrees to $\{0,1\}$ there are as many

\footnotetext{
*Departament de Llenguatges i Sistemes Informàtics, Universitat Politècnica de Catalunya, Jordi Girona 1-3, 08034 Barcelona, Spain. email address: anna.de.mier@upc.edu
} 
graphs with $(\operatorname{cros}(G)$, nest $(G))=(k, 1)$ as with $(\operatorname{cros}(G)$, nest $(G))=(1, k)$, although in this case the right degrees cannot be fixed. Computer assisted calculations show that the pair $(\operatorname{cros}(G)$, nest $(G))$ is symmetrically distributed over all graphs with $n \leq 10$ and $l_{i} \in\{0,1\}$ for $1 \leq i \leq n$; we believe that this is also the case for arbitrary $n$, but we have no proof of this fact. On the negative side, we give an example showing that if we consider arbitrary graphs, the pair $(\operatorname{cros}(G)$, nest $(G))$ is not symmetrically distributed. As explained later in Section 3, this counterexample answers on the negative a question of Krattenthaler on fillings of Ferrers diagrams.

\section{Graphs with left degree equal to 1}

For a subset $L \subset[n]$, let $\mathcal{G}_{L}$ be the set of graphs on $[n]$ where the left degree of vertex $i$ is 1 if $i \in L$ and 0 otherwise. (Note that $\mathcal{G}_{L}$ might be empty.) Within $\mathcal{G}_{L}$, there are as many 2 -noncrossing $k$-nonnesting as 2-nonnesting $k$-noncrossing graphs.

Theorem 1 For any integer $k \geq 1$ and any set $L \subset[n]$, there are as many graphs in $\mathcal{G}_{L}$ with $(\operatorname{cros}(G), \operatorname{nest}(G))=(k, 1)$ as with $(\operatorname{cros}(G)$, nest $(G))=(1, k)$.

Proof. We assume that $\mathcal{G}_{L}$ is nonempty since otherwise there is nothing to prove. We denote by $\mathcal{G}_{L, 1, \leq k}$ the set of graphs in $\mathcal{G}_{L}$ with $\operatorname{cros}(G)=1$ and nest $(G) \leq k$; similarly, $\mathcal{G}_{L, \leq k, 1}$ denotes the graphs in $\mathcal{G}_{L}$ with $\operatorname{cros}(G) \leq k$ and nest $(G)=1$. It is enough to show that $\left|\mathcal{G}_{L, 1, \leq k}\right|=\left|\mathcal{G}_{L, \leq k, 1}\right|$.

We define a bijection from $\mathcal{G}_{L, 1, \leq k}$ to $\mathcal{G}_{L, \leq k, 1}$ inductively on the number of elements of $L$. If $|L|=1$, then the only graph in $\mathcal{G}_{L}$ has two vertices and one edge, and belongs to both $\mathcal{G}_{L, 1, \leq k}$ and $\mathcal{G}_{L, \leq k, 1}$. Assume that we have bijections $\phi_{L^{\prime}}: \mathcal{G}_{L^{\prime}, 1, \leq k} \rightarrow \mathcal{G}_{L^{\prime}, \leq k, 1}$ for all $L^{\prime}$ with $\left|L^{\prime}\right|<l$. Now let $|L|=l$ and pick $G \in \mathcal{G}_{L, 1, \leq k}$. Let $x$ be the smallest element of $L$; since $G$ has no crossing and no isolated vertex, the neighbour of $x$ is $x-1$. Consider the graph $G^{\prime}$ obtained from $G$ by removing the edge incident with $x$ and all the isolated vertices resulting from this, if any; relabel the set of vertices $V\left(G^{\prime}\right)$ if necessary so that $V\left(G^{\prime}\right)=\left[\left|V\left(G^{\prime}\right)\right|\right]$. Let $L^{\prime}$ be the set of vertices of $G^{\prime}$ that have left degree equal to 1 ; let $H^{\prime}$ be the image of $G^{\prime}$ under $\phi_{L^{\prime}}$. We explain next how to complete $H^{\prime}$ to a graph $H$ in $\mathcal{G}_{L, \leq k, 1}$. It will be clear that the set of vertices with nonzero left degree in $H$ is $L$ and that nest $(H)=1$; after describing the construction we justify that $\operatorname{cros}(H) \leq k$. The transformation from $H^{\prime}$ into $H$ has 4 cases depending on the degrees of $x$ and $x-1$ in $G$.

(i) Suppose $r_{x}>0$ and $r_{x-1}>1$ in $G$. Then $H^{\prime}$ has $n$ vertices and $L^{\prime}=L-\{x\}$; obtain $H$ by adding the edge $\{1, x\}$ to $H^{\prime}$.

(ii) Suppose $r_{x}>0$ and $r_{x-1}=1$ in $G$. Then $H^{\prime}$ has $n-1$ vertices and $L^{\prime}=\{j-1 \mid j \in$ $L, j \neq x\}$. In this case to obtain $H$ relabel the vertices of $H^{\prime}$ to $\{2, \ldots, n\}$, add a vertex 1 and join $x$ to 1 .

(iii) Suppose $r_{x}=0$ and $r_{x-1}>1$ in $G$. Then again $H^{\prime}$ has $n-1$ vertices and $L^{\prime}=$ $\{j-1 \mid j \in L, j \neq x\}$. In this case we need to introduce a new vertex between the 
vertices $x-1$ and $x$ of $H^{\prime}$, then relabel the vertices correspondingly to $\{1,2, \ldots, n\}$, and then add the edge $\{1, x\}$.

(iv) Suppose $r_{x}=0$ and $r_{x-1}=1$ in $G$. Then $H^{\prime}$ ahs $n-2$ vertices and $L^{\prime}=\{j-2 \mid j \in$ $L, j \neq x\}$. Similarly, here we need to introduce two new vertices, one between vertices $x-2$ and $x-1$ of $H^{\prime}$ and another before vertex 1 . Then relabel the vertices and add the edge $\{1, x\}$.

We have to justify that this process does not create $(k+1)$-crossings in the graph $H$. By the inductive hypothesis, if there is a $(k+1)$-crossing in $H$ it must involve the new edge $\{1, x\}$; moreover, since $x$ is the first vertex with nonzero left degree, all edges in a $(k+1)$-crossing involving $\{1, x\}$ must have their left ends among $\{1,2, \ldots, x-1\}$. But since $G$ is in $\mathcal{G}_{L, 1, \leq k}$ and $x$ was the first element of $L$ and there are no isolated vertices, the vertices $1,2, \ldots, x-2$ are joined in $G$ to vertices $v_{1}, \ldots, v_{x-2}$, respectively, with the property that $v_{1}>v_{2}>\cdots>v_{x-2}$; this $x-2$ edges together with $\{x-1, x\}$ form an $(x-1)$-nesting in $G$, hence we must have that $x-1 \leq k$. So in $H$ we cannot have created a $(k+1)$-crossing by using an edge with a right-end in $x$, since there are at most $k$ vertices before it.

The inverse bijection $\mathcal{G}_{L, \leq k, 1} \rightarrow \mathcal{G}_{L, 1, \leq k}$ is defined in a similar manner and we omit the details.

\section{$3 \quad$ Arbitrary graphs}

We describe the example showing that the pair $(\operatorname{cros}(G)$, nest $(G))$ is not symmetrically distributed for arbitrary graphs in the language of fillings of Ferrers diagrams. We explain the correspondence between graphs and fillings of Ferrers diagrams after the statement of Fact 2 .

Given an integer partition $\lambda_{1} \geq \lambda_{2} \geq \cdots \geq \lambda_{s}$, the Ferrers diagram $F$ of shape $\left(\lambda_{1}, \ldots, \lambda_{s}\right)$ is the arrangement of square cells, from top to bottom and left-justified, with $\lambda_{i}$ cells in row $i$. A filling of $F$ consists of assigning a nonnegative integer to each cell of the diagram. If we restrict to the integers 0 and 1 we refer to 01 fillings to distinguish them from arbitrary fillings. A cell that has been assigned the integer 0 will be called empty. A se chain of length $k$ of the filling is a selection of rows $r_{1}<\cdots<r_{k}$ and columns $c_{1}<\cdots<c_{k}$ such that the cells $\left(r_{i}, c_{i}\right)$ are nonempty. A ne chain of length $k$ of the filling is a selection of rows $r_{1}<\cdots<r_{k}$ and columns $c_{1}<\cdots<c_{k}$ such that the cells $\left(r_{i}, c_{k-i+1}\right)$ are nonempty and with the additional condition that the cell $\left(r_{k}, c_{k}\right)$ is in the diagram.

We are interested in counting fillings where the sum of the entries is fixed. Let $N(F ; m ; n e=k, s e=l)$ be the number of arbitrary fillings of $F$ where the sum of the entries is $m$, the longest $n e$ chain has length $k$ and the longest $s e$ chain has length l. We add a superscript 01 to denote 01 fillings. It is known (see [2] or [3]) that $N(F ; m ; n e=k, s e=*)=N(F ; m ; n e=*, s e=k)$, where the $*$ indicates that there is no 
restriction in the corresponding parameter. It is actually shown that one can also fix the sums of the values of the filling along the rows and columns of the diagram. The same identity holds for 01 fillings, that is, $N^{01}(F ; m ; n e=k$, se $=*)=N^{01}(F ; m ; n e=*, s e=k)$, but in this case it is only possible to fix row sums (see [4], where this result follows from more general statements for moon polyominoes).

Krattenthaler [2, Problem 2] asks whether it is also the case that

$$
N^{01}(F ; m ; n e=t, s e=s)=N^{01}(F ; m ; n e=s, s e=t) .
$$

In his paper he shows that the equality holds when row and column sums are set to one. In [4] there are simple examples showing that if the claim is true then the restrictions on row and column sums must be dropped. We show that the answer to the problem is negative by giving a counterexample for the smallest possible values of $s$ and $t$.

Fact 2 For the Ferrers diagram $F$ of shape $(5,5,4,3)$,

$$
N^{01}(F ; 8 ; n e=1, s e=2)=10 \neq 11=N^{01}(F ; 8 ; n e=2, s e=1) .
$$

Before giving a proof of this fact, we make some remarks on the correspondence between graphs and fillings of Ferrers diagrams.

We consider graphs where each vertex has either left or right degree equal to zero; these graphs are called left-right graphs. A vertex with left degree (resp., right degree) equal to zero is called opening (resp., closing). There is an easy correspondence introduced in [3] between left-right graphs and fillings of Ferrers diagrams. Roughly speaking, to each left-right graph $G$ corresponds a filling $L(G)$ of a Ferrers diagram $F(G)$ whose shape is determined by the sequence of opening and closing vertices of $G$. The row sums of the filling $L(G)$ give the left degrees of $G$ and the column sums give the right degrees. Then $G$ has a $k$-crossing ( $k$-nesting) if and only if $L(G)$ contains a ne chain of length $k$ (se chain of length $k$ ). In this language, Theorem 1 above states that for all diagrams $F$ the equality $N^{01}(F ; m ; n e=1$, se $=k)=N^{01}(F ; m ; n e=k, s e=1)$ holds when restricted to fillings with row sums equal to 1.

In terms of graphs, Fact 2 translates as saying that for simple graphs on [9] with opening vertices $\{1,2,3,5,7\}$ and 8 edges in total, there are 10 with $(\operatorname{cros}(G)$, nest $(G))=$ $(1,2)$ and 11 with $(\operatorname{cros}(G)$, nest $(G))=(2,1)$. This does not show though that the pair $(\operatorname{cros}(G)$, nest $(G))$ is not symmetrically distributed over all simple graphs on [9] with 8 edges. To find such an example, we look at a simpler way to associate diagrams to graphs. This corresponds essentially to encoding the adjacency matrix of a graph on $[n]$ in the diagram $\Delta_{n}=(n-1, n-2, \ldots, 2,1)$ (this encoding was used by Krattenthaler in [2] to give alternative proofs of some of the results of [1]). We have that $N^{01}\left(\Delta_{n} ; m ; n e=k, s e=l\right)$ is the number of simple graphs $G$ on $[n]$ with $m$ edges and $(\operatorname{cros}(G)$, nest $(G))=(k, l)$. By an argument similar to the proof below of Fact 2 , it can be shown that $N^{01}\left(\Delta_{7} ; 11 ; n e=\right.$ $1, s e=2)=20 \neq 21=N^{01}\left(\Delta_{7} ; 11 ; n e=2\right.$, se $\left.=1\right)$.

Fact 2 above is for 01 fillings, but we can deduce from it a similar statement for arbitrary fillings. Note that the value of $N(F ; m ; n e=k, s e=l)$ can be deduced from 
the values of $N^{01}(F ; i ; n e=k, s e=l)$ for $1 \leq i \leq m$. The reason is that given a 01 filling of $F$ with $i$ nonempty entries, the number of ways of extending this filling to an arbitrary one with the same empty cells and with the sum of the entries equal to $m$ depends only on $i$. Hence it follows that for the smallest $m$ for which $N^{01}(F ; m ; n e=2$, se $=1) \neq$ $N^{01}(F ; m ; n e=1, s e=2)$ we must have that $N(F ; m ; n e=2, s e=1) \neq N(F ; m ; n e=$ $1, s e=2)$.

We now turn to the proof of Fact 2. The statement can be checked by computer, but for completeness we give a sketch of the counting by hand.

Proof of Fact 2.

Let $F$ be the diagram with shape $(5,5,4,3)$. First we show that $N^{01}(F ; 8 ; n e=1$, se = $2)=10$. Label the cells of $F$ as on the left hand-side of Figure 1. We distinguish several cases in the counting.

\begin{tabular}{|l|l|l|l|l|}
\hline $\boldsymbol{m}$ & $\boldsymbol{n}$ & $\boldsymbol{o}$ & $\boldsymbol{p}$ & $\boldsymbol{q}$ \\
\hline $\boldsymbol{h}$ & $\boldsymbol{i}$ & $j$ & $\boldsymbol{k}$ & $\boldsymbol{l}$ \\
\hline $\boldsymbol{d}$ & $\boldsymbol{e}$ & $\boldsymbol{f}$ & $\boldsymbol{g}$ & \multicolumn{1}{c}{} \\
\hline $\boldsymbol{a}$ & $\boldsymbol{b}$ & $\boldsymbol{c}$ & \multicolumn{1}{|c}{} \\
\cline { 1 - 3 } & & &
\end{tabular}

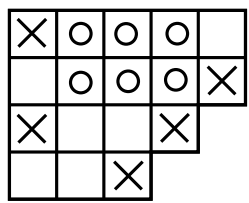

Figure 1: Figures used in the proof of $N^{01}(F ; 8 ; n e=1$, se $=2)=10$.

(i) Cell $d$ is in the filling. Then, since the maximum ne chain must have length one, we have that $i, j, k, n, o, p$ are empty. In total, we need 9 empty cells. To destroy the other ne-chains of length 2, each of the following pairs must contain an empty cell $\{h, q\},\{a, e\},\{b, f\}$. Hence $c, g, l, m$ are in the filling. The situation is depicted on the right hand-side of Figure 1, where $\times$ denotes a cell in the filling and $\circ$ denotes an empty cell. Now to avoid a se-chain of length 3 we have that $e$ must be empty as well, which forces $a$ to be in the filling and hence $f$ is empty and $b$ is in the filling. The last cell must be one of $\{h, q\}$ and since there are no more restrictions, this gives two possible fillings in this case.

(ii) Assume now that $d$ is empty and that $a$ is in the filling. By an argument analogous to the one in the previous case, we have that $e, f, i, j, n, o$ are empty and that $b, c, g, l, m$ are in the filling. The remaining two cells must be chosen one from each of the pairs $\{h, p\},\{k, q\}$. Of the four possible combinations, there is one that contains a nechain of length 2 , so there are three possibilities in this case.

(iii) If $d$ and $a$ are empty and $e$ is in the filling, then $j, k, o, p$ must be empty. Now we need 3 more empty cells but each of the four pairs $\{h, n\},\{i, q\},\{b, f\},\{m, c\}$ must contain an empty cell (the latter to avoid a se-chain of length 3). So there are no possible fillings in this case. 
(iv) If $a, d, e$ are empty and $b$ is in the filling, by an argument similar to that of case (i) we deduce that $f, h, i, j, o$ are empty and that $c, g, l, m, n, p$ are in the filling. Now the remaining cell in the filling can be either $k$ or $q$, so two possibilities.

(v) If $a, b, d, e$ are empty, by the same sort of argument as in the previous cases, we deduce that $h, i, j$ must also be empty.

(vi) Finally, if $a, b, d, e, i, j, h$ are all emtpy, then the remaining two empty cells must be one from each of the pairs $\{f, p\},\{k, q\}$. It is easy to see that three of the four possibilities give suitable fillings.

This shows that $N^{01}(F ; 8 ; n e=1, s e=2)=10$. The proof that $N^{01}(F ; 8 ; n e=$ 2$, se $=1)=11$ follows a similar argument. For completeness we list all possible fillings in Figure 2.
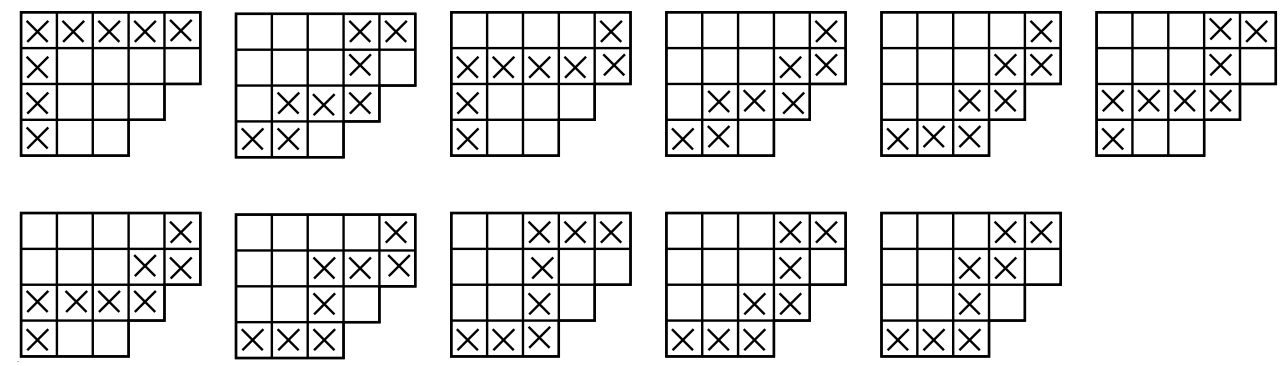

Figure 2: The 11 fillings of $(5,5,4,3)$ with $n e=2$ and $s e=1$

\section{References}

[1] W.Y.C. Chen, E.Y.P. Deng, R.R.X Du, R.P. Stanley, and C.H. Yan, Crossings and nestings of matchings and partitions, Trans. Amer. Math. Soc., to appear, arXiv:math.CO/0501230.

[2] C. Krattenthaler, Growth diagrams, and increasing and decreasing chains in fillins of Ferrers shapes, Adv. Appl. Math. 37 (2006), 404-431.

[3] A. de Mier, $k$-noncrossing and $k$-nonnesting graphs and fillings of Ferrers diagrams, arXiv:math.CO/0602195 v2.

[4] M. Rubey, Increasing and decreasing sequences in fillings of moon polyominoes, arXiv:math.CO/0604140. 